\title{
Plunging Ranula (Right side): A Case Report
}

\author{
Bardhan $\mathrm{A}^{1}$, Dev $\mathrm{PK}^{2}$, Banerjee $\mathrm{S}^{3}$, Islam $\mathrm{S}^{4}$
}

\begin{abstract}
Plunging ranulas, also known as deep, diving, cervical or deep plunging ranula, usually appear in conjunction with oral ranula. Rarely, these ranulas may arise independent of oral swelling. A rare case of plunging ranula without oral swelling is discussed along with review of literature.
\end{abstract}

\section{Introduction}

A plunging ranula is an extravasation of saliva from the sublingual gland due to trauma or obstruction of the duct.

Ranula can be divided into three types

*Oral or simple ranula

*Cervical or plunging ranula

*Mixed ranula

In case of plunging ranula fluid from the obstructed gland dissects between the fascial planes and muscles of the base of the tongue to the submandibular space. It is an uncommon variety. In most of the cases it is diagnosed clinically.

\section{Case Report}

A 23 yrs male reported with a 1.5 yrs history of swelling at Right submandibular region and rt side of the floor of the mouth (Figure $1 \& 2$ ). The swelling was completely asymptomatic and there was a history of intermittent change in size of swelling. The patient also gave history that there was no surgical intervention. But the swelling is gradually increasing in size for last 6 month for that region the patient visited at Jahurul Islam medical college Hospital at Otolaryngology out patient door.

\section{Discussion}

A ranula by definition is a mucus filled cavity, a mucocele, in the floor of the mouth in relation to the sublingual gland $^{1,2}$. The name "ranula" has been derived from the Latin word "rana" means "frog "ula" means little, which means little frog. The swelling resembles a frog's translucent

1. Corresponding Author: Dr. Ajoy Bardhan MBBS, DLO Assistant Professor, Department of Otolaryngology Jahurul Islam Medical College, Bajitpur, Kishoregonj

2. Dr. Prajit Kumar Dev MBBS, DLO Associate Professor, Department of Otolaryngology Jahurul Islam Medical College, Bajitpur, Kishoregonj

3. Dr. Sonjoy Banerjee MBBS, FCPS

Assistant Professor, Department of Otolaryngology Jahurul Islam Medical College, Bajitpur, Kishoregonj

4. Dr. Saiful Islam MBBS, DLO Assistant Professor, Department of Otolaryngology Jahurul Islam Medical College, Bajitpur, Kishoregonj

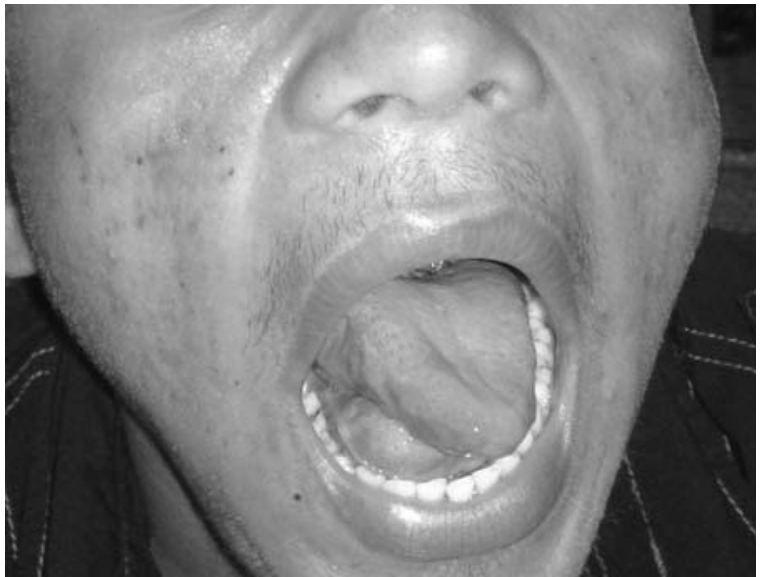

Fig. 1: Peroral View

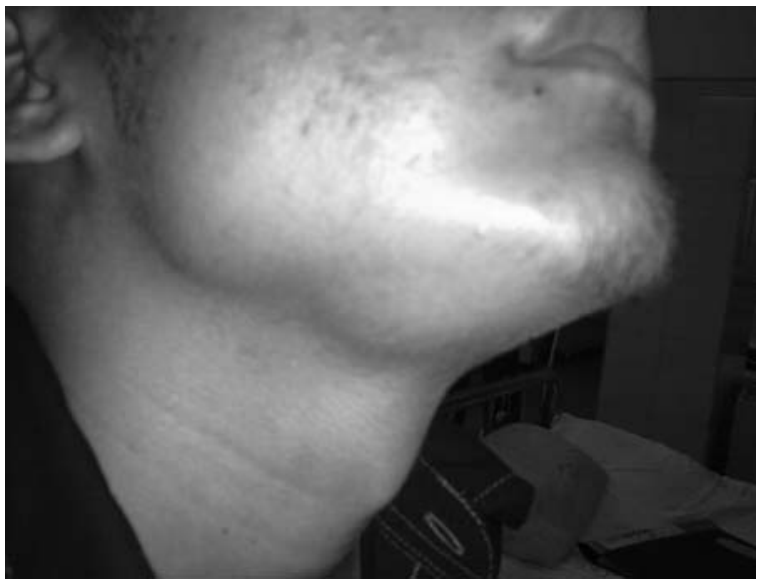

Fig. 2 : Submandibular View

underbelly or air sacs. Ranulas are characteristically large $(>2 \mathrm{~cm})$ and appear as a tense fluctuant dome-shaped vesicle, sometimes with a blue hue. The most common site is the lateral floor of the oral cavity. A clinical variant with moderate incidence, plunging ranula occurs when the fluid pressure of the mucin dissects through a perforation in the mylohyoid muscle in the submandibular space ${ }^{3-5}$. Ranulas have a prevalence of about 0.2 cases per 1000 persons and accounts for $6 \%$ of all oral sialocysts. The number of ranulas that represents a true retention cyst ranges from less than $1 \%$ to $10 \%$. Ranulas usually occur in children and young adults, with the peak frequency in the second decade ${ }^{6}$. The cervical variant tends to occur a little later in the third decade. The diagnosis of a plunging ranula is usually determined by a combination of history, clinical presentation, and imaging studies.

Plunging ranulas generally appear in conjunction with an oral ranula. Rarely can they arise independently of the oral 
component. In the absence of oral swelling, the clincal diagnosis of ranula may not be suspected. In upto $45 \%$ of the cases, the patient's first presentation is an oral swelling. Plunging ranulas are associated with oral swelling in $34 \%$ of cases. Another $21 \%$ of the cases occur without any oral involvement. The foremost etiology of ranulas is from the process such as partial obstruction of a sublingual duct. This can lead to formation of an epithelial-lined retention cyst, which occurs in $<10 \%$ of all ranulas. Second, most common factor is trauma that causes direct damage to the duct or deeper areas of the body of the sublingual gland, leading to extravasation of mucus and formation of pseudocyst. In most cases, it is iatrogenic. Plunging ranulas arise in the neck by one of the following four mechanisms. Firstly, the sublingual gland may project through the mylohyoid or an ectopic sublingual gland may exist on the cervical side of mylohyoid. This explains most plunging ranulas that exist without an oral component. Secondly, a dehiscence or hiatus in the mylohyoid muscle may occur. This defect is observed along the lateral aspect of the anterior two-thirds of the muscle. Through this defect, the mucin from the sublingual gland may penetrate to the submandibular space, Thirdly, approximately $45 \%$ of plunging ranulas occur iatrogenically after surgery to remove oral ranulas. Cases of plunging ranula formation have also been reported secondary to surgical procedures for sialolith removal, duct transposition and implant placement.Lastly, a duct from the sublingual gland may join the submandibular gland or its duct, allowing ranulas to from in continuity with the submandibular gland. Therefore, the ranula accesses the neck from behind the mylohyoid muscle ${ }^{4}$.

The cervical ranula appears as an asymptomatic, continuously enlarging mass that may fluctuate in size. Most reported ranulas are $4-10 \mathrm{~cm}$ in size. The overlying skin is usually intact. The mass is fluctuant,freely movable, and nontender. The mass is not associated with the thyroid gland or lymph node chains. In some instances, detecting salivary gland herniation of a portion of the sublingual gland through the mylohyoid muscle into the neck may be possible. The mass may not be well defined but follow the fascial planes of the neck and may extend into the mediastinum. Similar to the oral ranula, the mass tends to cause a lateral swelling; however, it may cross the midline. They have been reported to extend into the submental region, the contralateral neck, the nasopharynx upto the skull base, the retropharynx and even into the upper mediastinum ${ }^{7,8}$. Rarely, large-sized ranulas may cause dysphagia or airway obstruction. In our case, the patient presented with a swelling that was restricted to right submandibular region and the patient was completely asymptomatic. On injecting a contrast medium in the sublingual space, we could appreciate the extension of the dye in right submandibular space, which was suggestive of a plunging ranula. although, Ultresonography is usually nondiagnostic, the USG for this patient showed a cystic mass at right submandibular region.

Though the cases of plunging ranula have been documented with moderate frequency, failure to differntiate the clinical features of oral and plunging ranulas may be a diagnostic pitfall. these lesions may be difficult to differentiate from benign and malignant salivary gland tumors, especially cystadenocarcinoma and mucoepidermoid carcinoma. A case of squamous cell carcinoma in the wall of ranula has also been reported ${ }^{9}$. so thorough radiological, biochemical, and histopathological investigations should be carried out for all case of suspected plunging ranulas.

\section{References}

1. Baurmash HD. Mucoceles and ranulas. Journal of Oral and Maxillofacial Surgery. 2003;61:369-378. [PubMed]

2. Neville BD, Damm DD, Allen CM, Bouquot JE. Oral and Maxillofacial Pathology. 2nd edition. Philadelphia, Pa, USA: Saunders;2002.

3. Langlois NEI, Kolhe P. Plunging ranula: a case report and a literature review. Human Pathology. 1992;23:1306-1308. [PubMed]

4. de Visscher JGAM, van der Wal KGH, de Vogel PL. The plunging ranula. Pathogenesis, diagnosis and management. Journal of Cranio-Maxillo-Facial Surgery. 1989;17:182-185. [PubMed]

5. Skouteris CA, Sotereanos GC. Plunging ranula: report of a case. Journal of Oral and Maxillofacial Surgery. 1987;45:1068-1072. [PubMed]

6. Zhao Y-F, Jia Y, Chen X-M, Zhang W-F. Clinical review of 580 ranulas. Oral Surgery, Oral Medicine, Oral Pathology, Oral Radiology and Endodontology. 2004;98:281-287. [PubMed]

7. Horiguchi H, Kakuta S, Nagumo M. Bilateral plunging ranula. A case report. International Journal of Oral and Maxillofacial Surgery. 1995;24:174-175. [PubMed]

8. Shelley MJ, Yeung KH, Bowley NB, Sneddon KJ. A rare case of an extensive plunging ranula: discussion of imaging, diagnosis, and management. Oral Surgery, Oral Medicine, Oral Pathology, Oral Radiology, and Endodontics. 2002;93:743-746. [PubMed]

9. Ali MK, Chiancone G, Knox GW. Squamous cell carcinoma arising in a plunging ranula. Journal of Oral and Maxillofacial Surgery. 1990;48:305-308. [PubMed] 